\title{
Nonfactorizable charm-loop effects in exclusive FCNC B-decays
}

\section{Dmitri Melikhov*}

D. V. Skobeltsyn Institute of Nuclear Physics, M. V. Lomonosov Moscow State University, 119991, Moscow, Russia, and

Institute for High Energy Physics, Austrian Academy of Sciences, Nikolsdorfergasse 18, A-1050

Vienna, Austria, and

Faculty of Physics, University of Vienna, Boltzmanngasse 5, A-1090 Vienna, Austria

E-mail: dmitri_melikhov@gmx.de

\section{Anastasiia Kozachuk}

D. V. Skobeltsyn Institute of Nuclear Physics, M. V. Lomonosov Moscow State University, 119991, Moscow, Russia

adkozachukegmail.com

This talk presents our recent results [四] on nonfactorizable charm-loop contributions to the amplitudes of rare exclusive $B$-decays induced by flavour-changing neutral currents (FCNC). We show that such contributions induced by a soft-gluon emission from the charm-quark loop may be expressed via three-particle quark-antiquark-gluon distribution amplitude (3DA) of the $B$ meson, $\left\langle 0\left|\bar{q}(x) G_{\mu v}(y) b(0)\right| B(p)\right\rangle$, and the knowledge of the full functional dependence of the latter quantity on the variable $(x-y)^{2}$ is necessary for a proper summation of potentially large terms $O\left(\left(\Lambda_{Q C D} m_{b} / m_{c}^{2}\right)^{n}\right)$ in the amplitudes of FCNC $B$-decays.

European Physical Society conference on High Energy Physics - EPS-HEP 2019,

10-17 July 2019

Ghent, Belgium

\footnotetext{
*Speaker.
} 


\section{Introduction}

The interest in charm-loop contributions to rare FCNC decays of the $B$-mesons is to the large extent motivated by the fact that virtual charm-quark loops, including charmonia states which appear in the physical region of the $B$-decay, may have a strong impact on the $B$-decay observables [D] thus providing a "noise" for the extraction of possible new physics effects.

It is known that in the charmonia region, nonfactorizable gluon exchanges dominate the amplitudes. A number of theoretical analyses of nonfactorizable effects induced by charm-quark contributions has been published in the literature: an effective gluon-photon local operator describing the charm-quark loop has been calculated in [B] for the real photon as an expansion in inverse charm-quark mass $m_{c}$ and applied to inclusive $B \rightarrow X_{s} \gamma$ decays; Ref. [四] obtained a nonlocal effective gluon-photon operator for the virtual photon and applied it to inclusive $B \rightarrow X_{s} l^{+} l^{-}$decays. In [5] nonfactorizable corrections in exclusive FCNC $B \rightarrow K^{*} \gamma$ decays using local OPE have been studied; in [ [6, प], these corrections have been analyzed with light-cone sum rules using local OPE for the photon-gluon operator and three-particle light-cone distribution amplitudes of $K^{*}$-meson.

As emphasized in [ [3], $6, \mathbb{B},-[0]$, local OPE for the charm-quark loop leads to a power series in $\Lambda_{\mathrm{QCD}} m_{b} / m_{c}^{2}$. This parameter is of order unity for the physical masses of $c$ - and $b$-quarks and thus corrections of this type require resummation. The authors of [ए]] derived a different form of the nonlocal photon-gluon operator compared to [ [ 4 ] and evaluated its effect at small values of $q^{2}$ ( $q$ momentum of the lepton pair) making use of light-cone 3-particle DA (3DA) of the $B$-meson with the aligned arguments, $\left\langle 0\left|\bar{s}(y) G_{\mu v}(u y) b(0)\right| B_{s}(p)\right\rangle$.

In a recent work [U] we have emphasized that the full consistent resummation of $\left(\Lambda_{\mathrm{QCD}} m_{b} / m_{c}^{2}\right)^{n}$ terms requires the generic 3DA of the $B$-meson, $\left\langle 0\left|\bar{s}(y) G_{\mu \nu}(x) b(0)\right| B_{s}(p)\right\rangle$, with non-aligned coordinates.

We illustrated this result by analyzing a field theory with scalar quarks/gluons; the generalization to QCD is straightforward. The counting scheme in which the parameter $\Lambda_{\mathrm{QCD}} m_{b} / m_{c}^{2}$ is kept of order unity was adopted.

\section{Toy model: Nonfactorizable corrections in a field theory with scalar particles}

To avoid technical complication related to a Lorentz/spinorial structure of the amplitudes, we discuss nonfactorizable effects of interest for spinless particles. We nevertheless use the QCD notations for quark fields and assume the following hierarchy of quark masses

$$
m_{b} \gg m_{c} \gg m_{s} \sim \Lambda_{\mathrm{QCD}}
$$

whereas the parameter $\Lambda_{\mathrm{QCD}} m_{b} / m_{c}^{2}$ is assumed to be of order unity,

$$
\Lambda_{\mathrm{QCD}} m_{b} / m_{c}^{2} \sim 1
$$

The charm contribution to the amplitude of an FCNC $B$-decay reads

$$
A(p, q)=i \int d z e^{i q z}\left\langle 0\left|T\left\{c^{\dagger}(z) c(z), s^{\dagger}(0) s(0)\right\}\right| B_{s}(p\rangle,\right.
$$

where quark fields are understood as Heisenberg field operators in a theory that involves weak and strong interactions. 


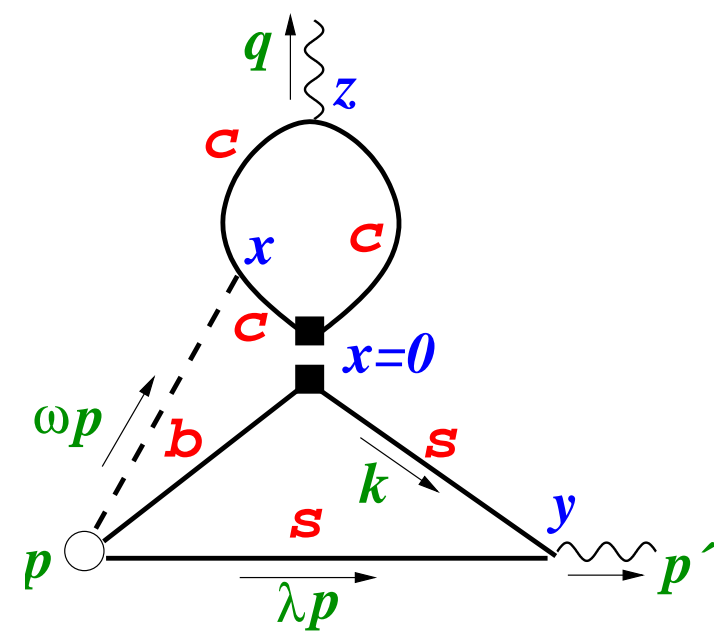

Figure 1: One of the diagrams describing the nonfactorizable gluon exchange. Dashed line corresponds to gluon; $q$ and $\kappa=-\omega p$ are the momenta outgoing from the charm-quark loop; the momentum $q^{\prime}=q+\kappa=$ $q-\omega p$ is emitted from the $b \rightarrow s$ vertex. Another diagram, equal to the one shown in the figure, corresponds to the Gluon attached to the right $c$-quark line in the upper loop.

Our goal is to study nonfactorizable corrections due to a soft-gluon exchange between the charm-quark loop and the $B$-meson loop. To lowest order, the corresponding amplitude is given by the diagram of Fig. W:

$$
A(p, q)=i \int d z e^{i q z}\left\langle 0\left|T\left\{c^{\dagger}(z) c(z), i \int d y^{\prime} L_{\text {weak }}\left(y^{\prime}\right), i \int d x L_{G c c}(x), s^{\dagger}(0) s(0)\right\}\right| B_{s}(p\rangle .\right.
$$

The effective Lagrangian that mimics weak four-quark interaction is chosen in a simple form

$$
L_{\text {weak }}=\frac{G_{F}}{\sqrt{2}} s^{\dagger} b c^{\dagger} c,
$$

while the interaction of the scalar gluon field $G(x)$ and the scalar $c$-quarks is taken to be

$$
L_{\mathrm{Gcc}}=G(x) c^{\dagger}(x) c(x) .
$$

\subsection{Charm-quark loop with soft gluon emission is perturbative}

The charm contribution is described by the three-point function. For our analysis it is important, that in the region $q^{2} \ll 4 m_{c}^{2}, q^{\prime 2}$ and $\kappa^{2}$ are also far below the charm thresholds, i.e. the charm loop is perturbative. To show that, we use the gluon field in momentum representation, which is related to the gluon field in coordinate representation as follows

$$
G(x)=\frac{1}{(2 \pi)^{4}} \int d \kappa \tilde{G}(\kappa) e^{i \kappa x}, \quad \tilde{G}(\kappa)=\int d x G(x) e^{-i \kappa x} .
$$

Then the effective operator describing the gluon emission from the charm quark loop may be written as

$$
\mathscr{O}(q)=\int d \kappa \tilde{G}(\kappa) \Gamma_{c c}(\kappa, q),
$$


where $\Gamma_{c c}(\kappa, q)$ stands for the contribution of two triangle diagram with the charm quark running in the loop. The momenta $\kappa$ and $q$ are outgoing from the charm-quark loop, whereas the momentum $q^{\prime}=q+\kappa$ is emitted from the $b \rightarrow s$ vertex. $p^{\prime}$ is the momentum of the outgoing $s^{\dagger} s$ current and $p$ is the momentum of the $B$-meson, $p=p^{\prime}+q$.

In terms of the gluon field operator in coordinate space, we can rewrite (2.8) as

$$
\mathscr{O}(q)=\int d \kappa e^{-i \kappa x} d x G(x) \Gamma_{c c}(\kappa, q)
$$

\subsection{Three-particle antiquark-quark-gluon distribution amplitude of $B$-meson}

By virtue of ([.9), the amplitude Eq. ([2.4) takes the form

$$
A(q, p)=\frac{1}{(2 \pi)^{8}} \int \frac{d k}{m_{s}^{2}-k^{2}} \int d y e^{-i\left(k-p^{\prime}\right) y} \int d x e^{-i \kappa x} d \kappa \Gamma_{c c}(\kappa, q)\left\langle 0|\bar{s}(y) G(x) b(0)| B_{s}(p) \nvdash 2.10\right)
$$

Here, we encounter the $B$-meson three-particle amplitude with three (non-aligned) arguments, for which one may write down the following decomposition:

$$
\left\langle 0\left|s^{\dagger}(y) G(x) b(0)\right| B_{s}(p)\right\rangle=\int d \lambda e^{-i \lambda y p} \int d \omega e^{-i \omega x p}\left[\Phi(\lambda, \omega)+O\left(x^{2}, y^{2},(x-y)^{2}\right)\right] .
$$

Here $\lambda$ and $\omega$ are dimensionless variables. Making use of the properties of Feynman diagrams, one may show that they should run from 0 to 1 . However, if one of the meson constituents is heavy, it carries the major fraction of the meson momentum and as the result the function $\Phi(\lambda, \omega)$ is strongly peaked in the region

$$
\lambda, \omega=O\left(\Lambda_{\mathrm{QCD}} / m_{b}\right)
$$

So, effectively one can run the $\omega$ and $\lambda$ integrals from 0 to $\infty$; such integration limits in fact emerge in the DAs within heavy-quark effective theory [एव, [2]. We emphasize that for the results presented below only peaking of the DAs in the region ([2.2) is essential. Notice also that the function $\Phi(\lambda, \omega)$ in (L. I) coincides with the same function that appears in the "standard" 3particle distribution amplitude with the aligned arguments, $x=u y$, discussed in [ए2].

\subsection{Light-cone contribution}

First, let us calculate the contribution to $A(q, p)$ from the term that corresponds to $x^{2}=y^{2}=$

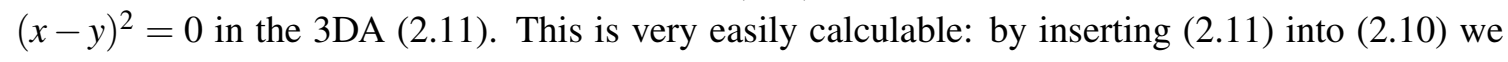
can perform the $x$ - and $y$-integrals

$$
\begin{aligned}
& \int d x \rightarrow \delta(\kappa+\omega p), \\
& \int d y \rightarrow \delta\left(k+\lambda p-p^{\prime}\right) .
\end{aligned}
$$

Next, the $\delta$-functions above kill the integrals over $k$ and $\kappa$, and we find

$$
A(q, p)=\int_{0}^{\infty} d \lambda \int_{0}^{\infty} d \omega \Phi(\lambda, \omega) \Gamma_{c c}(-\omega p, q) \frac{1}{m_{s}^{2}-\left(\lambda p-p^{\prime}\right)^{2}} .
$$


As the result one obtains for the sum of two triangle diagrams (with the charm quark running in the loop in two opposite directions) the following expression for the amplitude:

$$
\Gamma_{c c}(\kappa, q)=\frac{1}{8 \pi^{2}} \int_{0}^{1} d u \int_{0}^{1-u} d v \frac{1}{m_{c}^{2}-2 u v \kappa q-u(1-u) \kappa^{2}-v(1-v) q^{2}} .
$$

Now, we must take into account that the $\omega$-integral is peaked at $\omega \sim \Lambda_{\mathrm{QCD}} / m_{b}$ so the gluon is soft: $\kappa=-\omega p$ and $\kappa^{2} \sim O\left(\Lambda_{\mathrm{QCD}}^{2}\right) \ll m_{c}^{2}$.

\section{$2.4 s$-quark produced in a weak $b \rightarrow s$ transition is highly virtual}

The momentum transferred in the weak-vertex is $q^{\prime}=q+\kappa=q-\omega p$, such that

$$
q^{\prime 2}=(q-\omega p)^{2}=q^{2}-\omega(1-\omega) M_{B}^{2}-q^{2} \omega+p^{\prime 2} \omega=q^{2}-\omega(1-\omega) M_{B}^{2} .
$$

By virtue of the $y$-integration in (2.13]), the $s$-quark propagator takes the form

$$
m_{s}^{2}-\left(\lambda p-p^{\prime}\right)^{2}=m_{s}^{2}-\lambda q^{2}+(1-\lambda)\left(\lambda M_{B}^{2}-p^{\prime 2}\right) .
$$

Therefore, in the bulk of the $\lambda$-integration the virtuality of the $s$-quark propagator is large, $O\left(M_{B}\right)$, and the use of the Feynman $s$-quark propagator is well justified.

\subsection{Deviations from the light-cone}

We now turn to the calculation of the contributions to $A(q, p)$ generated by terms $\sim x^{2}, y^{2},(x-$ $y)^{2}$ in the 3DA (‥JI). The terms containing powers of 4 -vectors $y$ and $x$ in the integral (2.10) can be calculated by parts integration. Taking into account the results $([2.13)$, we find the following relative contributions of the terms containing different powers of the coordinate variables:

$$
\begin{aligned}
\Lambda_{\mathrm{QCD}}^{2} y^{2} & \rightarrow \frac{\Lambda_{\mathrm{QCD}}}{m_{b}}, \\
\Lambda_{\mathrm{QCD}}^{2} x^{2} & \rightarrow \frac{\Lambda_{\mathrm{QCD}}^{2}}{m_{c}^{2}} \frac{\Lambda_{\mathrm{QCD}} m_{b}}{m_{c}^{2}}, \\
\Lambda_{\mathrm{QCD}}^{2} x y & \rightarrow \frac{\Lambda_{\mathrm{QCD}} m_{b}}{m_{c}^{2}} .
\end{aligned}
$$

Clearly, all terms containing powers of $x^{2}$ and/or $y^{2}$ in the 3DA lead to the suppressed contributions to $A(q, p)$ and may be neglected within the considered accuracy. However, the terms containing powers of $x y$ lead to the contributions containing powers of $\Lambda_{\mathrm{QCD}} m_{b} / m_{c}^{2}$, i. e., of order unity within the adopted counting rules. The kinematical configurations that give the dominant contribution to the amplitude is thus rather simple: the vectors $x$ and $y$ are directed along the light-cone [e.g., $x$ along the $(+)$ axis, and $y$ along the $(-)$ axis], but the 4-vector $(x-y)$ is obviously not directed along the light cone. Therefore, the full dependence of 3DA on the variable $(x-y)^{2}$ is needed in order to properly resum corrections of order $\left(\Lambda_{\mathrm{QCD}} m_{b} / m_{c}^{2}\right)^{n}$. 


\section{Conclusions}

The relevant object that arises in the calculation of the nonfactorizable corrections is the threeparticle DA (or the antiquark-quark-gluon vertex function of the $B$-meson):

$$
\left\langle 0\left|s^{\dagger}(y) G(x) b(0)\right| B_{s}(p)\right\rangle=\int d \lambda e^{-i \lambda y p} \int d \omega e^{-i \omega x p}\left[\Phi(\omega, \lambda)+O\left(x^{2}, y^{2},(x-y)^{2}\right)\right] .
$$

It should be emphasized that the function $\Phi(\omega, \lambda)$ here is precisely the same function that parameterizes the standard 3DA with the aligned arguments, $x=u y$, on the light cone $x^{2}=0$, discussed in [ㅁ] ]. At small $q^{2} \leq m_{c}^{2}$, terms of order $\sim x^{2}, y^{2}$ yield the suppressed contributions to the nonfactorizable amplitude of $B$-decay compared to the contribution of the light-cone term in the three-particle DA: for terms $O\left(x^{2}\right)$ the suppression parameter is $\Lambda_{\mathrm{QCD}}^{2} / m_{c}^{2}$, and for terms $O\left(y^{2}\right)$ the suppression parameter is $\Lambda_{\mathrm{QCD}} / m_{b}$. However, terms $\sim(x y)^{n}$ in the 3DA yield potentially large contributions to the $B$-meson amplitude, of order $\left(\Lambda_{\mathrm{QCD}} m_{b} / m_{c}^{2}\right)^{n}$. The resummation of such contributions requires the knowledge of the full dependence of $\left\langle 0\left|s^{\dagger}(y) G(x) b(0)\right| B_{s}(p)\right\rangle$ on its variable $(x-y)^{2}$.

We emphasize that the knowledge of merely the light-cone 3DA, corresponding to $x^{2}=0$, $y^{2}=0$ and $(x-y)^{2}=0$, is not sufficient: While the LC 3DA resums a part of the large corrections of the order $\Lambda_{\mathrm{QCD}} m_{b} / m_{C}^{2}$, other contributions of the same order remain unaccounted.

\section{Acknowledgments}

D. M. is grateful for support to the Austrian Science Fund FWF under Project P29028 and to RFBR under joint CNRS/RFBR Grant 19-52-15022. A. K. was supported by Grant No.16-1210280 of the Russian Science Foundation.

\section{References}

[1] A. Kozachuk and D. Melikhov, Revisiting nonfactorizable charm-loop effects in exclusive FCNC B-decays, Phys. Lett. B786, 378 (2018).

[2] M. Beneke, G. Buchalla, M. Neubert, and C. T. Sachrajda, Penguins with Charm and Quark-Hadron Duality, Eur. Phys. J. C61, 439 (2009).

[3] M. B. Voloshin, Large $O\left(m_{c}^{-2}\right)$ nonperturbative correction to the inclusive rate of the decay $B \rightarrow X_{s} \gamma$, Phys. Lett. B397, 275 (1997).

[4] G. Buchalla, G. Isidori, and S. J. Rey, Corrections of order $\Lambda_{\mathrm{QCD}}^{2} / m_{c}^{2}$ to inclusive rare B decays, Nucl. Phys. B511, 594 (1998).

[5] A. Khodjamirian, R. Ruckl, G. Stoll, and D. Wyler, QCD estimate of the long distance effect in $B \rightarrow K^{*} \gamma$, Phys. Lett. B402, 167 (1997).

[6] P. Ball and R. Zwicky, Time-dependent CP Asymmetry in $B \rightarrow K^{*} \gamma$ as a (Quasi) Null Test of the Standard Model, Phys. Lett. B642, 478 (2006).

[7] J. Lyon and R. Zwicky, Resonances gone topsy turvy - the charm of QCD or new physics in $b \rightarrow s l^{+} l^{-}$? arXiv:1406.0566.

[8] Z. Ligeti, L. Randall, and M. B. Wise, Comment on nonperturbative effects in $\bar{B} \rightarrow X_{s} \gamma$, Phys. Lett. B402 178 (1997). 
[9] M. Benzke, S. J. Lee, M. Neubert, G. Paz, Factorization at Subleading Power and Irreducible Uncertainties in $\bar{B} \rightarrow X_{s} \gamma$, JHEP 1008, 099 (2010).

[10] A. Khodjamirian, T. Mannel, A. Pivovarov, and Y.-M. Wang, Charm-loop effect in $B \rightarrow K^{(*)} l^{+} l^{-}$ and $B \rightarrow K^{*} \gamma$, JHEP 1009, 089 (2010).

[11] A. Kozachuk, D. Melikhov, N. Nikitin, Rare FCNC radiative leptonic $B_{s, d} \rightarrow \gamma l^{+} l^{-}$decays in the Standard Model, Phys. Rev. D 97, 053007 (2018).

[12] H. Kawamura, J. Kodaira, C.-F. Qiao, and K. Tanaka, B-meson light cone distribution amplitudes in the heavy quark limit, Phys. Lett. B523, 111 (2001), Erratum: Phys. Lett. B536, 344 (2002). 